\title{
HIGHLIGHTS
}

ANTIPLATELET THERAPY

\section{Long-term dual antiplatelet therapy and bleeding in stable patients-insights from CHARISMA}

The long-term risks and benefits of dual therapy with aspirin and clopidogrel are now better known owing to the results of the CHARISMA trial.

"As new and more potent oral antiplatelet agents become available, it is critical to understand the risks and benefits of the existing agents," says CHARISMA investigator Peter Berger. Most previous studies have assessed the risks of dual antiplatelet therapy, in particular the risk of bleeding, in the acute setting. Little was known about long-term risks of clopidogrel use among patients with stable vascular disease.

In the CHARISMA trial, patients with stable vascular disease or with risk factors for vascular disease were randomly assigned to receive clopidogrel or placebo in addition to aspirin therapy. Patients were followed for a median 28 months and bleeding events were assessed according to the GUSTO (Global Utilization of Streptokinase and t-PA for Occluded Coronary Arteries) criteria.
Clopidogrel, compared with placebo, was associated with an increased risk of severe (1.7\% versus $1.3 \%$ patients) and moderate $(2.1 \%$ versus $1.3 \%$ patients) bleeding. Importantly, although the risk of bleeding was increased in the first year of clopidogrel use, this difference was no longer observed after the first year of therapy in patients who had not experienced moderate or severe bleeding.

Moderate bleeding was strongly associated with mortality. The risks of bleeding did not differ between patients with established stable disease and patients with risk factors only. Therefore, the increased mortality seen among patients with risk factors alone compared with patients with established stable disease is not explained by an increased bleeding risk. "This analysis sheds light on why clopidogrel was not found to be useful in patients with risk factors for vascular disease but without demonstrable vascular disease," says Berger.

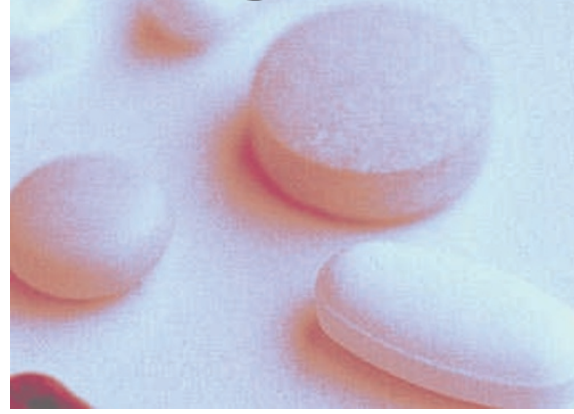

Similar long-term studies of other antiplatelet agents will be useful to guide therapeutic decisions. Berger and colleagues are currently studying the risks and benefits of other antiplatelet drugs in patients unable to take clopidogrel.

Joana Osório

Original article Berger, P. B. et al. Bleeding complications with dual antiplatelet therapy among patients with stable vascular disease or risk factors for vascular disease: results from the Clopidogrel for High Atherothrombotic Risk and Ischemic Stabilization, Management and Avoidance (CHARISMA) trial. Circulation 121, 2575-2583 (2010) 